\title{
Implementing residency programs and its challenges
}

\section{Authors}

Chong Chia Yin ${ }^{1,2,3 *}$; Oh Jean Yin'; Bernard Chern Su Min'; Serene A.K. Ong4; Yeo Ai Ling ${ }^{4}$; Chay Oh Moh"1,2,3

\section{Abstract}

The exponential growth of Singapore's population from the influx of migrants over recent years, coupled with an aging population, has resulted in a need to increase the number of doctors and, in addition, raise their expertise. The British-based system of specialist training was inherited by Singapore and it consisted of two time-based phases: Basic Specialist Training and Advanced Specialist Training. However, in June 2010, the United States-based Residency system was adapted because it was curriculum-based with a more structured framework. This change in systems has resulted in a number of problems such as: (a) additional faculty to train; (b) adequate manpower backfill; (c) adequate resources for residents, including new infrastructure to facilitate learning, and (d) the usual anxiety associated with such a major change, especially from those who did not see the need to change the current system.

Since manpower backfill was a key issue, overseas specialist staff and non-specialist doctors were recruited to facilitate protected training time for residents. In addition, there was a redesign of service work for healthcare personnel. Although these solutions have helped to alleviate many of the problems, the challenge facing the program now is its sustainability since it has proven to be resource intensive.

\section{Keywords}

residency; ACGME international; pediatrics; postgraduate medical education; curriculum reform

\section{Introduction}

Singapore's population increase ${ }^{1}$ has highlighted a need not only to increase the current number of doctors, but to raise their expertise, as well. The former is achieved through increasing student intakes, setting up a third medical school, and recruiting foreign doctors. The latter is addressed by a review of postgraduate medical education, where Singapore recently transitioned from the British-based specialist training system to the United States-based residency system. ${ }^{2,3}$

In this article, the challenges and problems faced in establishing and accrediting residency programs in the setting of a busy hospital, KK Women's and
'KK Women's and Children's Hospital, Singapore

${ }^{2}$ National University of Singapore (NUS) Yong Loo Lin School of Medicine, Singapore

${ }^{3}$ Duke-NUS Graduate Medical School, Singapore.

${ }^{4}$ Academy, Group Education, Singapore Health Services Pte. Ltd, Singapore.

* E-mail: Chay.oh.moh@kkh.com.sg.

\section{Cite this article as:}

Yin CC, Yin OJ, Min BCS, Ong SAK, Ling YA, Moh CO. Implementing residency programs and its challenges.. Innovations in Global Health Professions Education. 2015:5. http://dx.doi.org/10.20421/ighpe2015.5

This is an open access article distributed under the terms of the Creative Commons Attribution license CC BY 4.0, which permits unrestricted use, distribution and reproduction in any medium, provided the original work is properly cited. 
Children's Hospital (KKH), are reviewed. Solutions employed, as well as local adaptations, are also discussed.

\section{KK Women's and Children's Hospital}

$\mathrm{KKH}$ is Singapore's only integrated hospital for women and children, and one of the largest children's hospital in the region. $\mathrm{KKH}$, originally known as Kandang Kerbau Hospital, opened in 1924 with 30 beds and 12 children's cots and catered mainly to women's healthcare, particularly obstetrics and gynecology (O\&G). In 1997, KK Hospital was renamed KK Women's and Children's Hospital, reflecting its expanded role in both O\&G and Pediatrics. Today, the 830-bed hospital is a Joint Commission International accredited hospital and a tertiary referral center for the nation regarding high risk obstetrical cases, state-of-the art gynecological evaluation and treatment, pediatrics, and life-saving neonatology.

$\mathrm{KKH}$ has seen a steady increase in workload yearly; by $2009, \mathrm{KKH}$ was averaging 170,000 in Children's Emergency attendance (with an average of 450 per day), 22,000 inpatient admissions, 100,000 outpatient visits, and 600 Pediatric Intensive Care Unit and 1400 Neonatal Intensive Care Unit admissions. However, manpower was limited, with only 71 full-time specialist staff and three part-time specialist staff, a result of the Ministry of Health's (MOH) policy to restrict the number of sponsored training positions to six per year in pediatrics.

\section{Comparing old and new systems}

Singapore inherited a United Kingdom-based system of specialist training, which consisted of two phases: Basic Specialty Training (BST), and Advanced Specialty Training (AST). This system of specialist training involved skill-based clinical training and a summative examination of two to three years of BST, followed by three years of AST. Progression in training was time-based with an emphasis on the summative examination, consisting of either the specialist Member of the Royal College of Pediatricians and Child Health (MRCPCH), or the local Master of Medicine (M.Med) Pediatrics examination. Learning was opportunistic and dependent on the motivation of learners and faculty. This system came under tremendous stress in institutions with limited manpower situations, in which patient care was naturally prioritized and education placed on the back burner.

Since July 2010, MOH has started a new United States-based residency system. In the United States, residency programs are accredited by the Accreditation Council for Graduate Medical Education (ACGME), and international graduate medical education programs are accredited by ACGMEinternational, or ACGME-I. The residency model has a structured training framework and a curriculum based on the six core competencies: patient care, medical knowledge, practice-based learning and improvement, interpersonal and communication skills, professionalism, and systems-based practice. One notable difference with this new system are the regular formative evaluations, which ensure a continual review of the learner's progress (Figure 1).

In the previous system, trainees underwent sixmonthly rotations, which may have taken place in different hospitals, but under the residency program, residents now stay with the Sponsoring Institution (SI) for at least three years. This provides an opportunity for a continuous curriculum and supervision.

In the past, education was embedded in a doctor's clinical role, and clinicians were expected to carry out clinical duties and teach simultaneously (Figure 1). However, ACGME advocated adequate support for an environment conducive to learning, for residents as well as faculty. Therefore, a better balance is struck between education and clinical duties.

$\mathrm{MOH}$ implemented the new residency program with aims to improve training of doctors in both acute and primary care settings, promote postgraduate medical education as integral to the professional development of a doctor, and to cope with the anticipated surge in postgraduate trainees.

\section{Problems encountered with the implementation of the new system}

The problems encountered can be separated into three categories: (a) general concerns faced with implementation of change to existing systems; (b) problems specific to $\mathrm{KKH}$, and (c) additional local requirements. 
OLD

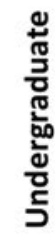

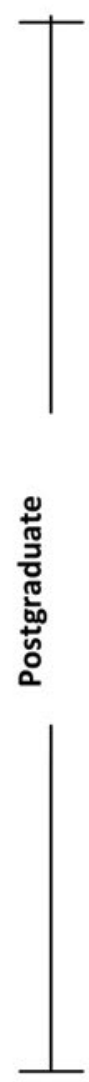

NEW

5 years

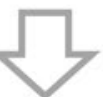

House Officer (HO)/Internship

1 year

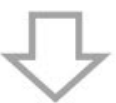

Basic Specialty Training (BST)

3 years

Two exams: M.Med and/or MRCPCH

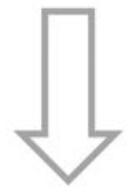

Advanced Specialty Training (AST)

$2-3$ years
MBBS

5 years

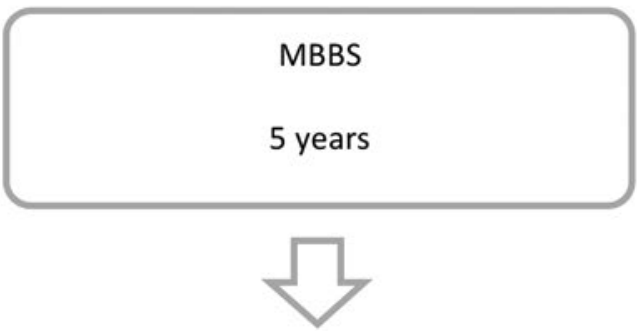

Licensing requirements

6-8 months

Residency

3 years

Three formative exams: annual ITE

Two summative exams: M.Med \& PME

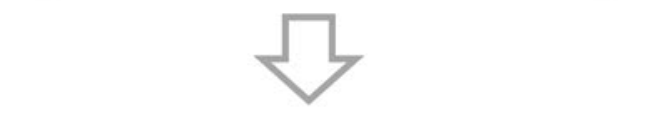

Senior Residency

$2-3$ years

MBBS: (Bachelor of Medicine, Bachelor of Surgery); M.Med: Masters of Medicine; MRCPCH: Member of the Royal College of Paediatricians and Child Health; ITE: InTraining Exams; PME: Paediatric Medicine Examination

Figure 1. Pediatrics residency program compared with the previous program 


\section{General concerns}

Difficulties encountered with the implementation of the new residency system included: (a) additional faculty to train; (b) adequate manpower backfill; (c) adequate resources for residents, including new infrastructure to facilitate learning, and (d) the usual anxiety associated with a major change, especially from those who did not see the need to change the current system.

There was already a shortage of staff, with many institutions having unfilled positions. Furthermore, ACGME-I requirements stipulate a ratio of one core faculty to six residents for residency, and a ratio of one faculty to two residents for senior residency, and that educators and residents are both given protected time for education. Thus, recruitment of overseas specialist staff, particularly those with a strong interest in education, would be necessary. At the same time, extra personnel to help take over part of the service load, would also have to be recruited. In the past, trainees functioned as any other intern or medical officer. Now under the ACGME framework, residents play the role of learners rather than clinical staff, thus junior staff had to be recruited to assist with the service load of residents.

In addition, an environment conducive to learning, a place for residents to interact, adequate learning resources, and accessibility to updated information, which translates to easy computer access, are ACGME requirements that were either not present or lacking in most institutions. Outpatient clinics with strategic templates for planned appointments to allow residents to clerk, examine, and interact with faculty were also necessary. Hence, more space was required for educational purposes.

This venture has proven to be resource and cost intensive. The challenges facing the program are in its capacity of meeting all the requirements from ACGME, and its sustainability in the long term.

In the interim period where there were significant numbers of inflight trainees from the previous system, the issues of fairness and equitability were important. Trainees felt that they had been marginalized and feared that they would be made to assume additional workload from the residents. In addition, as the intake of residents was not subjected to a mandatory cap and that number was expected to increase, the trainees were worried about competition with residents for resources.

\section{Problems specific to KKH}

The decision to change the postgraduate training program in 2009 came at a time in which the institution was facing a severe manpower shortage and dealing with a surge in attrition, as new private practice groups pulled specialists away from the hospital. $\mathrm{KKH}$ is a hospital primarily focused on clinical service and is also a member of SingHealth, Singapore's largest healthcare provider, which had the largest number of trainees and fellows, and would naturally be the most affected by the proposed changes.

Pediatrics was one of the first five specialties chosen to launch the new residency programs, and the institution had to prepare for external accreditation by ACGME-I. Furthermore, an additional program, O\&G Residency, was to be launched the following year. KKH is a hospital primarily focused on clinical service, and both teaching faculty and trainees have a heavy clinical workload and service commitment. Hence, many staff and faculty members were unable to devote additional time and effort to the program.

As an institution focused primarily on clinical care, there was a need to have a mindset change to view education as one of the key pillars for the hospital's growth and development plan. Furthermore, $\mathrm{KKH}$ was heavily committed to supporting undergraduate medical, nursing, and allied health education. $\mathrm{KKH}$ had to provide the capacity to meet the needs of the other SI-sponsored programs, such as Pediatric Emergency Medicine, Pediatric and Gynecological Pathology, Women's Psychiatry, Pediatric Surgery, and Family Medicine, despite struggling to meet internal institutional needs for our anchor programs of Pediatrics and O\&G.

\section{Additional local requirements}

The United States residency format was only adapted and not wholly adopted; certain past requirements were still maintained. For instance, for the Pediatric program, all first year residents have to complete an additional eight months of broadbased training in Internal Medicine and General 
Surgery before starting Pediatric rotations, thus the local Pediatric program is $\mathbf{4 4}$ months instead of $\mathbf{3 6}$ months long.

In addition to the annual In-Training Exams (ITE) in Pediatrics, which are required as part of a United States accredited program, residents also need to pass the M.Med (Pediatrics) and MRCPCH exams. Furthermore, unlike the United States residency program, which allows residents who have completed three years of residency to be Board certified and to practice independently as community pediatricians or hospitalists, local residents have to undergo an additional three years of Senior Residency and pass an exit examination before they are certified as a specialist pediatrician by the Specialist Accreditation Board (SAB) in Singapore. SAB does not have an equivalent board certification after three years of residency; therefore even if residents are certain that they only wanted to be community pediatricians, they have no alternative except to complete further training before they can leave for independent practice in child health. This is an anomaly in the Singapore system, whereby after one year of internship after undergraduate medical school, a doctor can leave for independent practice to treat adults and children as a General Practitioner. Conversely, a Pediatric resident who has completed three years of structured training in Pediatrics is not recognized as a competent community pediatrician.

The O\&G Residency program, on the other hand, is a four-year ACGME-I accredited program, instead of the usual three years in ACGME. Residents undergo an annual in-training exam, called the Council on Resident Education in Obstetrics and Gynecology (O\&G) examination. Residents are also required to complete an intermediate examination and in addition, a postgraduate examination to complete their residencies.

\section{Resources allocated}

Funding and resources were pledged by $\mathrm{MOH}$ to help set up these new residency programs. Funding and resources were used to provide (a) new infrastructure to facilitate learning, (b) adequate manpower backfill to allow service staff time for training, (c) additional faculty to train residents, and (d) to engage the team from the United States ACGME-I to provide training to the Designated
Institutional Officer (DIO), Assistant Designated Institutional Officer (ADIO), Program Directors (PD), Program Coordinators (PC), and faculty.

Hardware, such as computers, was easy to procure, but space was a constraint in $\mathrm{KKH}$. Despite additional funding for backfill, the problem of finding skilled staff remained. Funds were also used to train faculty in mentoring, giving feedback, and making standardized evaluations.

\section{Solutions}

The DIO oversees all Residency programs, and the ADIO position was created to facilitate coordination of Residency program affairs in $\mathrm{KKH}$. A trip to the annual ACGME Conference in 2009 was helpful in learning about the structure, framework of supervision and evaluation, and the concepts of competency-based formative evaluation. Visits were also organized to a few residency centers in the United States to learn about the logistics of organizing residency programs.

As $\mathrm{KKH}$ prepared for accreditation, foreign specialists were brought in, particularly those with a strong interest in pedagogy. It was also valuable to recruit specialists who had been immersed in residency programs during their training. They brought with them the knowledge and contributed their personal experiences regarding the ACGME Residency program. In addition, non-specialist doctors were recruited to help maintain the service load to facilitate protected training time for residents. Moreover, swift action was necessary, and resources were needed, even before funding from $\mathrm{MOH}$ came in.

In consultation with $\mathrm{MOH}, \mathrm{KKH}$ advocated for the redesign of service work of healthcare personnel. It was proposed that tasks such as routine phlebotomy and intravenous cannulation be taken over by patient care assistants. The nursing team rendered support in hiring and training phlebotomists and venous access teams. In addition, with the Medical Board's approval, experienced nurses were specially selected and appointed and trained to take over some of the roles of junior residents. After a month of lectures and four months of training, these nurses could clerk, examine, and perform simple procedures for 
three common conditions in patients above three years of age: gastroenteritis, upper respiratory tract infections, and urinary tract infections. These Resident Nurses became critical partners in ensuring protected learning time for residents, and in maintaining a high standard of clinical care. This example also highlights the need for strong support from all involved. The advice "ensure that people are on board and on your side" 4 was often echoed by others involved in ambitious programs of change.

Examining the new curriculum, two of the six core competencies (practice-based learning and improvement, and system-based practice) were identified as new. To help in teaching the core competencies, a comprehensive training program was constructed, which was useful for the PD, who prepared the curriculum, rotations, evaluation tools, and Program Information Form.

KKH's strong collaboration with Duke-NUS on undergraduate medical training helped to build a strong relationship with Duke University in the United States. It was with the help of many individuals previously involved in residency programs that we managed to reduce the obstacles of this transitional journey.

The ADIO met with residents frequently to listen to their challenges. This was critical for the initial period since they were highly anxious about this new program. It was through these interactions that gaps were identified and solutions explored. For example, residents were concerned about long night duties, which followed the $24+6$ rule (continuous on-site duty not exceeding 24 hours, and may remain for up to six additional hours for didactic activities, transfer care of patients, and to conduct outpatient clinics). This resulted in excessive fatigue and residents needed time to recover from call, thus limiting time for self-study. Residents were encouraged to explore alternatives, including the United States night float system. Eventually, O\&G adopted the night float system while Pediatric residents negotiated to pilot a 24-hour night call system. In the past, doctors on call stayed on duty beyond 24 hours, averaging 30-36 hours per call. Now, the on-call resident will hand over clinical duties the following morning and leave at 24 hours. This prevented fatigue in doctors, which translated to improved patient care during the nightshift.

\section{Conclusion}

Lam and Lam 5 noted in a survey of Asian countries that reformed medical education in Asia, though based on Western models, is adapted locally to the cultural and community needs. Singapore has put together a modified residency program, certified by ACGME-I but with local content and additional ministry requirements. Since Singapore is the first country outside of the United States to achieve successful accreditation of residency programs, our experience may serve as a blueprint for other hospitals interested in implementing similar postgraduate medical education programs.

As a new hybrid program, further changes are fully expected. Although these solutions have helped to alleviate many problems, the challenge now is its sustainability. One continuing challenge faced by the DIO and ADIO is helping the PD, faculty, and residents comprehend, understand, come to terms with, and prepare for changes ahead. It is also the role of the $\mathrm{DIO}$ and ADIO to provide feedback and advocate for the best learning experience for residents, who are our pipeline in providing sustainable, high quality, and affordable care.

Acknowledgements: It was the timely assistance of Professor Katheryn Adoloseck of the Graduate Medical Education office, Duke University Health System, that helped us navigate the complexity of the Institutional requirements.

The authors are also grateful to Dr. Colin Song (past DIO), A/Prof Lim Boon Leng (current DIO)and A/ Prof Goh Siang Hiong (ADIO from Changi General Hospital) for their support and guidance.

Disclosures: No funding or financial support was received for this manuscript. Ethical approval was not applicable, and this work had not been previously presented.

\section{References}

1. Singapore Department of Statistics, Ministry of Trade and Industry [Internet]. Census of Population 2010 Statistical Release 1. Demographic characteristics, education, language and religion [cited 2015 Nov 30]. Available from http://www.singstat.gov. 
sg/publications/publications-and-papers/ cop2010/census10_stat_release1.

2. Lim BL. Residency programmes in Singapore-Challenges ahead. Ann Acad Med Singapore. 2013;42:265-266.

3. Huggan PJ, Samarasekara DD, Archuleta S, Khoo SM, Sim SHS, Sin CSP, et al. The successful, rapid transition to a new model of graduate medical education in Singapore. Acad Med. 2012;87:1268-1273.

4. Pritchard LS. Changing course. Med Educ. 2004;38:582-586.

5. Lam TP, Lam YYB. Medical education reform: The Asian experience. Acad Med. 2009;84(9):1313-1317. 